\title{
Model Identification for an Active Vibration Isolator
}

\author{
WANG Yun Feng ${ }^{1, a^{*}}$, ZHAO Bao Shan ${ }^{1, b}$ and WANG Li Tong ${ }^{1, c}$ \\ ${ }^{1}$ Tianjin Institute of Aerospace Mechanical and Electrical Equipment, China \\ ªvangyunfengdoc@126.com, bbszhaohit@126.com, cwanglitong21@126.com
}

\begin{abstract}
Keywords: active vibration isolator, model identification, model reduction, NR-LMS.
Abstract. In this article, the process model of an active vibration isolator including the secondary path and the primary path are developed based on state space theory and NR-LMS identification method. The frequency response functions from the identified model and the experiment are compared, The results indicate this identification method is useful to build structural model and the model developed is accurate to describe real structures dynamic characters.
\end{abstract}

\section{Introduction}

Reaction wheel assembly (RWA) and control moment gyroscopes (CMGs) used for attitude control and pointing at scientific targets are the primary disturbance sources which degrade the pointing precision and camera's image quality ${ }^{[1,2]}$. The primary feature of these disturbances is that they have very small amplitude of vibration, but the degradation of image quality caused by them is serious. Therefore attenuating these vibrations would enhance the performance of the optical payloads effectively. The vibration isolation is a feasible way and there have been many researchers provided their methods for vibration attenuation ${ }^{[3-7]}$. In the active vibration isolation system the active controller is indispensible. While the modern control methods being used to design the controller, the dynamic model of controlled structure must be known. The dynamic model based on identification methods is effective and accurate for control application ${ }^{[8]}$.

The state space model has been widely used in modern control theory, and the Eigensystem Realization Algorithm (ERA) is a typical algorithm to identify dynamic system's model ${ }^{[9]}$. Cao Y. ${ }^{[10]}$ employs the Maximum A Posteriori (MAP) algorithm to identify the model of 3 degree of freedom piezoelectric stage, and experiment are carried on to validate the identified model. De Callafon R. A. ${ }^{[11]}$ achieves structure's modal parameters by useing the General Realization Algorithm (GRA) to identify structural state space model, and the statistical properties of the modal parameter provided by the GRA are investigated through numerical simulation based on a benchmark problem with non-classical damping.

In this paper, an active vibration isolator with 8-input and 8-output is proposed with its control model (secondary path model) and disturbance model (primary path model) being identified by employing the state space theory and NR-LMS algorithm proposed in [12]. The transfer function of the identified model is compared with that from experiment, and the conclusion is given out in the end.

\section{Model of Active Vibration Isolator}

The active vibration isolator (AVI) is presented in Fig 1, it contains a load board, four piezoelectric folded-beams, and a base. The disturbance source, especially the RWA, is fixed on the load board. Piezoelectric folded beams with piezoelectric patches glued on their surfaces as sensors and actuators are orthogonal to each other, and they are the primary components of control platform.

This AVI can measure the vibration level of disturbances from the source and generate control forces to attenuate vibration under the commands of controller. The base is the connector between the platform and the plant (satellite or spacecraft). In Fig 1(a), the vertical elements are normal space beam elements, the horizontal elements are piezoelectric beam elements which have piezoelectric layers glued on their upper and lower surfaces. The actuator and the sensor on the same beam have the same number and they are a actuator/sensor pair. The 3D model and the real active isolator are shown in Fig 
1 (b) and Fig 1 (c) respectively. All the numbers of actuator/sensor pairs corresponding to the numbers of beams are shown in Tab. 1.

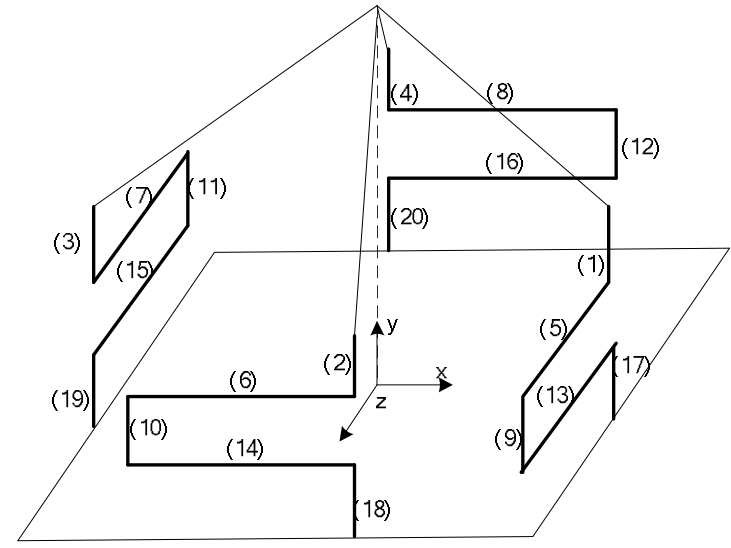

( a )

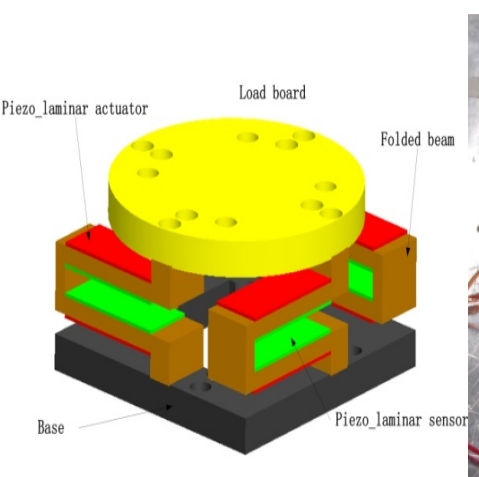

( b )

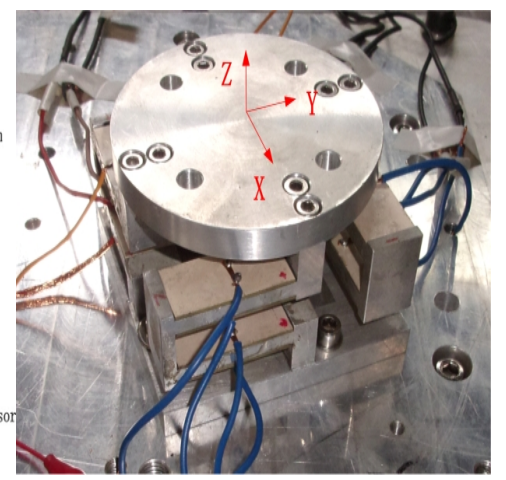

( c )

Fig 1 The active isolator model .(a) sketch map of AVI, (b) 3D model, (c) the real active isolator

Table 1 Numbers of actuator/sensor pairs corresponding to beam

\begin{tabular}{ccccccccc}
\hline Object & \multicolumn{8}{c}{ number } \\
\hline $\begin{array}{c}\text { Actuator/sensor } \\
\text { pair }\end{array}$ & 1 & 2 & 3 & 4 & 5 & 6 & 7 & 8 \\
Beam & 5 & 6 & 7 & 8 & 13 & 14 & 15 & 16 \\
\hline
\end{tabular}

\section{Model Identification}

Reference [1] has studied the control performance of this AVI by simulation employing its finite element model and POF controller under the conditions of random disturbance. A good process model is often required for designing a good controller. The controller based on a good process model often works better than the ones designed without a model. However, model development from the first principles is a difficult and expensive task, it is also a time consuming and tedious process ${ }^{[13]}$. The FEM models are sometimes not feasible for control process because the order should be high for achieving the desired accuracy. The system identification technique based upon the experimentation offers a rather simplistic approach for getting the process model of the system. By using the input applied to the system and the output signals a model can be achieved.

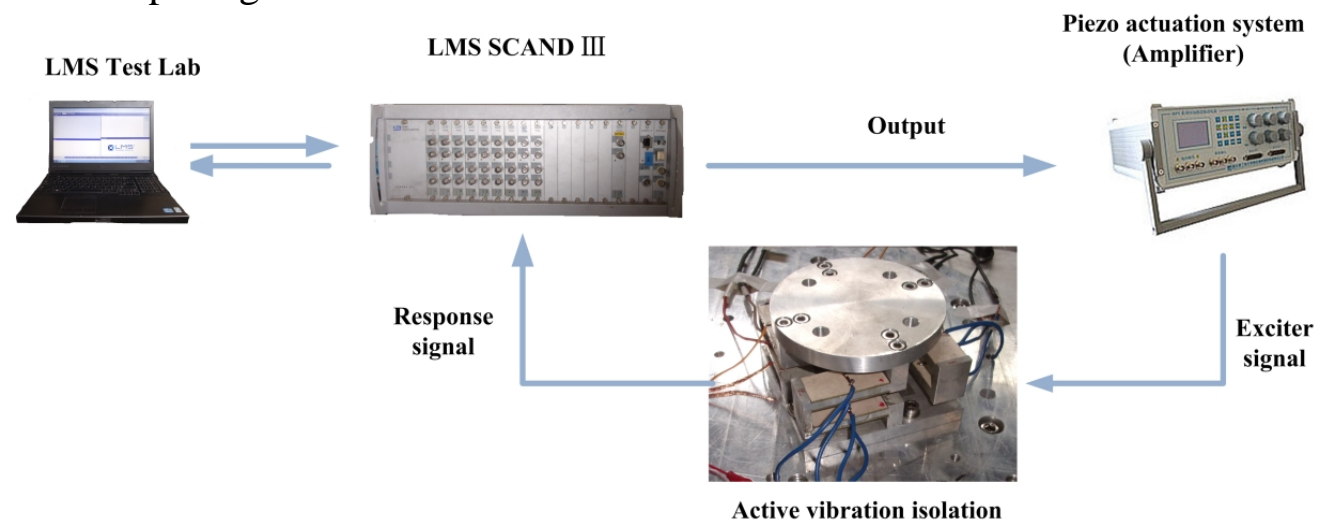

Fig 2 The flow chart of identification experiment

The identification experiment process is shown as Fig 2. To identify the secondary path model (control model) of the AVI, the AVI system needs to be stimulated via the eight actuators. We use a group of chirp signals sent out from the LMS SCAND $\square$ and amplified by the Piezo-actuation System as the input to one actuator. The same experimental processes are repeated eight times with one actuator being stimulated at a time. In the LMS test Lab, we set the frequency range of chirp signals being $0-1024 \mathrm{~Hz}$ and its amplitude being 1 . The time of sampling is $16 \mathrm{~s}$ and the sample points gathered is 16384. In every individual experimental process, the output signals from the eight sensors are 
captured by LMS SCAND $\square$, and all the input and output signals are used to identify the model. The identification method proposed in reference ${ }^{[12]}$ are employed here, and eight single-in-eight-out state space models are obtained with their order being 14. The NR-LMS algorithm is given as

$$
\begin{gathered}
\boldsymbol{y}(n)=\boldsymbol{W}^{T}(n) \boldsymbol{u}(n) \\
\boldsymbol{e}(n)=\boldsymbol{d}(n)-\boldsymbol{y}(n) \\
\boldsymbol{W}(n+1)=\boldsymbol{W}(n)+\mu\left[\frac{\gamma_{1} \boldsymbol{u}(n) \boldsymbol{e}(n)}{\|\boldsymbol{u}(n)\|^{2}}+\frac{\gamma_{2} \boldsymbol{u}(n-1) \boldsymbol{e}(n-1)}{\|\boldsymbol{u}(n-1)\|^{2}}+\frac{\gamma_{3} \boldsymbol{u}(n-2) \boldsymbol{e}(n-2)}{\|\boldsymbol{u}(n-2)\|^{2}}\right]
\end{gathered}
$$

where $\boldsymbol{e}(n-1)=\boldsymbol{d}(n-1)-\boldsymbol{y}(n-1)$ and $\boldsymbol{e}(n-2)=\boldsymbol{d}(n-2)-\boldsymbol{y}(n-2)$. The coefficients $\gamma_{1}, \gamma_{2}$ and $\gamma_{3}$ are constrained as

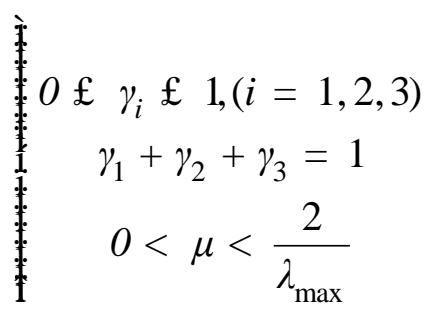

The eight state space models are assembled with the same sequence as the actuator/sensor numbers, and the complete control model's order is 112 with 8 inputs and 8 outputs. We assume the complete model is equal to the real AVI system here. For getting good control performance, model reduction is often performed ${ }^{[14]}$. But the reduction would degrade the accuracy of the model, further the control performance would be affected. Here based upon the system model's Hankel matrix, we define the model error $e_{r}$ is

$$
e_{r}=\frac{\mathbf{H}_{a}-\mathbf{H}_{r}}{\mathbf{H}_{a}}
$$

where, $\mathbf{H}_{r}$ is the sum of all the Hankel singular values of the reduced model, $\mathbf{H}_{a}$ is the sum of all the Hankel singular values of the complete model. After reduction, the order of new model is 64 with model error being 0.4\%. All the Hankel singular values are represented in Fig 3. By further reduction, we reserve the first 30 Hankel singular values and get a 30 order model with model error $2 \%$.

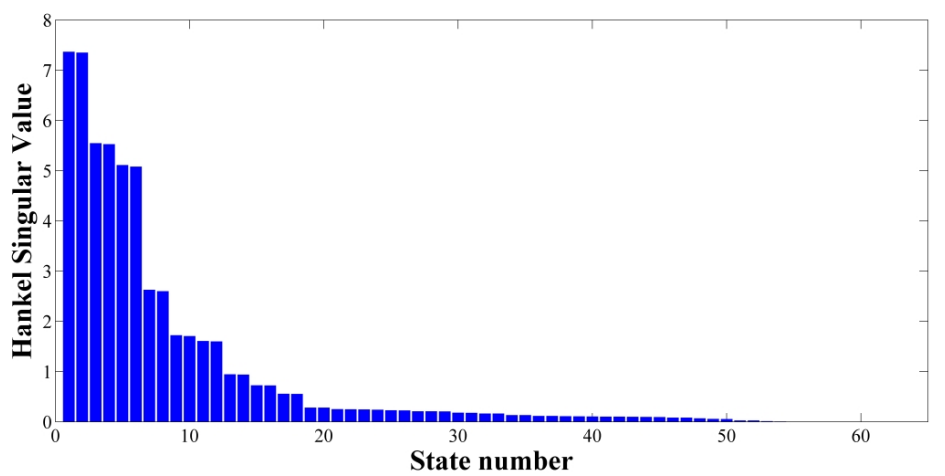

Fig 3 Hankel singular values of the reduced control model

For validating the reduced model, same chirp signals are inputted to the 5-th actuator of the 30 order model and the 64 order model respectively, and their output signals are collected. The transfer functions from the reduced model and the experimental measurement are compared here. Fig 4 displays the transfer function from sensor 1 and sensor 5 to actuator 5 of the 64 order model and the experimental data respectively, and the same transfer function of 30 order model is displayed in Fig 5. By comparative analysis, we can find out both the models match closely with the real AVI system, and the 64 order model matches better than the 30 order model because of its higher order and less model error. 

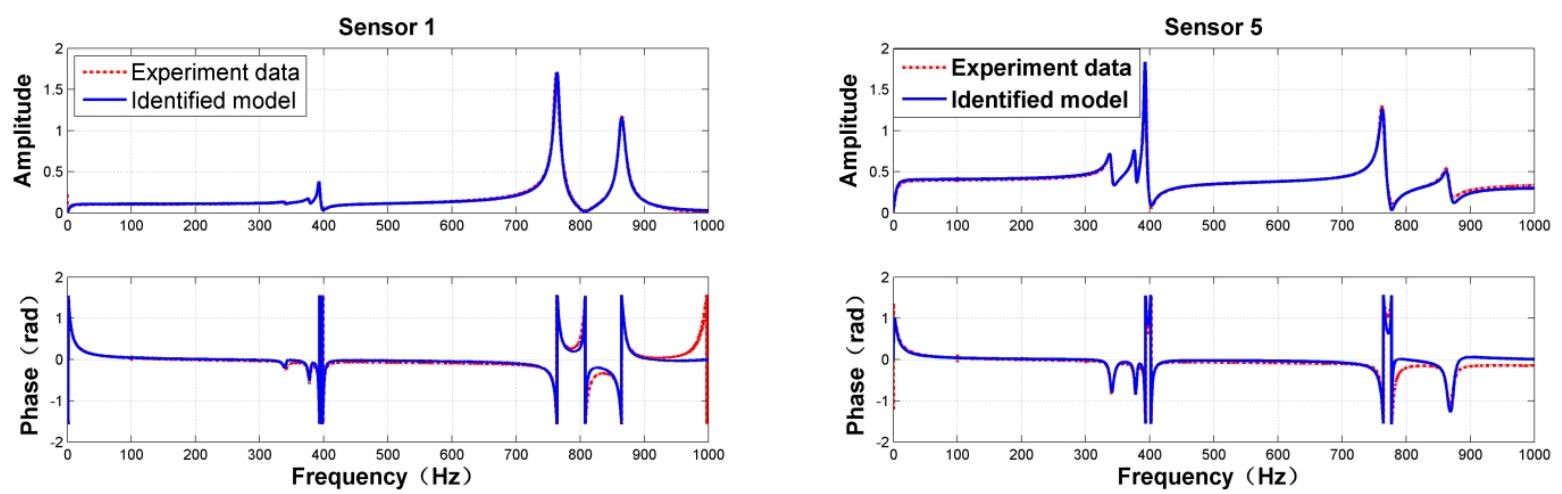

Fig 4 The transfer functions of the 64 order control model and the real AVI
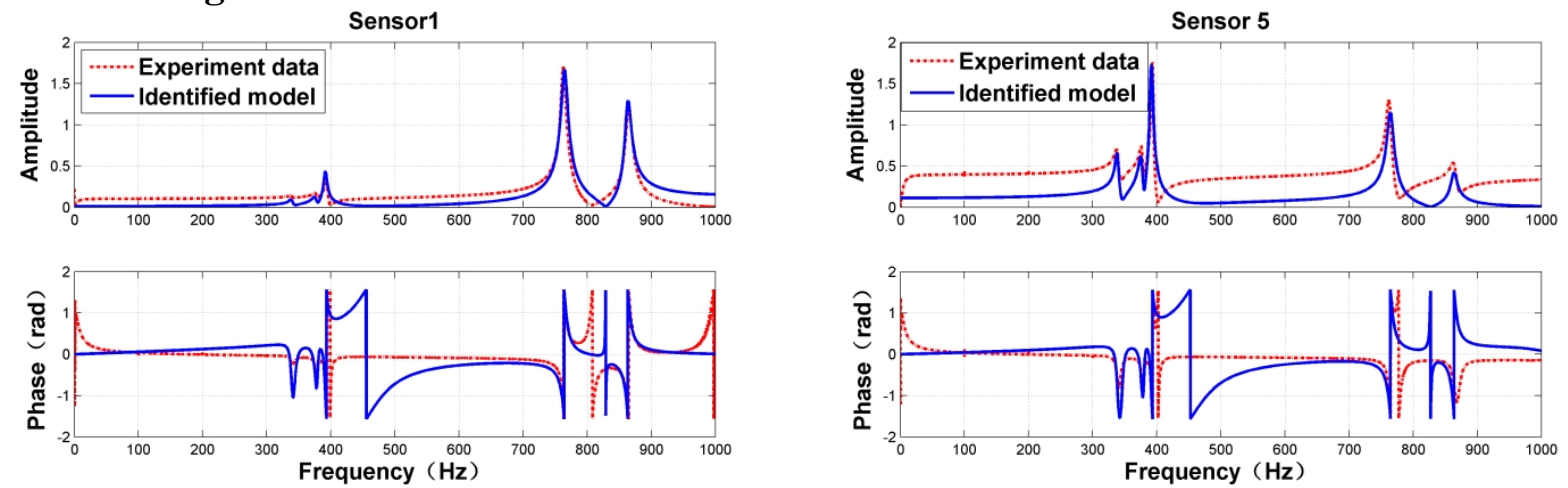

Fig 5 The transfer functions of the 30 order control model and the real AVI

The disturbance model (primary path model), which describes the dynamics from the disturbance to the sensors of AVI, must be identified for the control simulation. Its identification process is the same as control model. In the experiment, force hammer is used as disturbance exciter to tap the AVI at the load board edge along the $\mathrm{X}, \mathrm{Y}$ and $-\mathrm{Z}$ directions respectively once at a time. The force signals and outputs of the AVI are collected by LMS SCAND $\square$, and we get three groups of data after this experiment. Each of the data groups are used for identification and three single-in-eight-out models are achieved. The order of all the three models is eight, and after assemblage according to the sequence $\mathrm{X}-\mathrm{Y}-\mathrm{Z}$, we get the complete 24 order disturbance model. For simplifying the calculation, model reduction is performed too, and after adjusting the order of disturbance model is 10 with the model error less than $3 \%$ relative to the 24 order model. The same as the validation of control model, we also use the transfer function from the experimental data and the reduced model to be compared. Fig 6 displays the transfer function from sensor 1 and sensor 5 to disturbance along X-direction of the reduced disturbance model and the experimental data respectively, and it is clear the reduced model covers the primary characteristics of the disturbance model (primary path) and it has enough accuracy for the usage of control simulation.
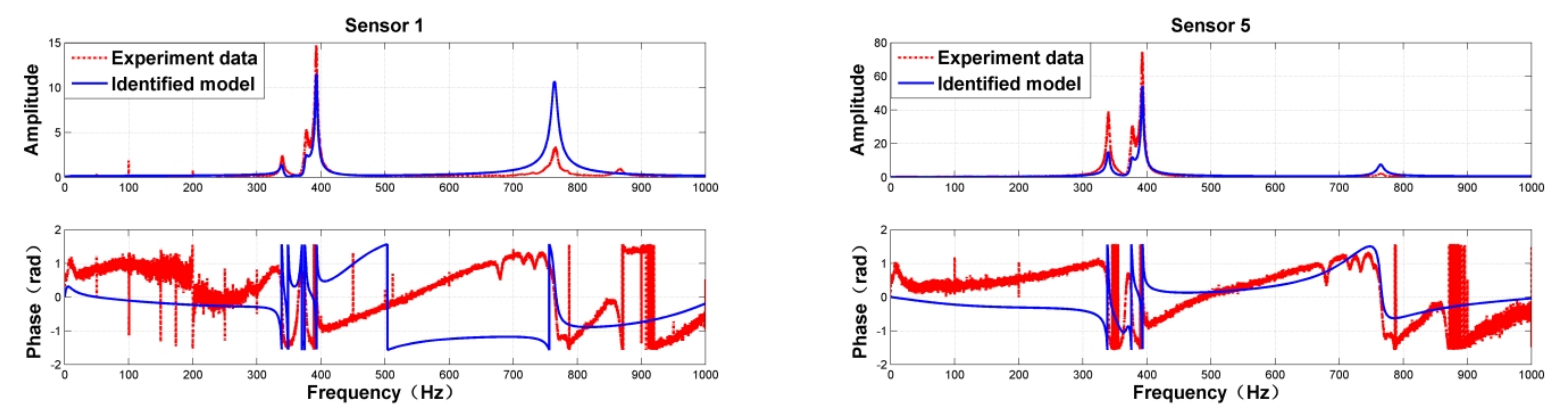

Fig 6 The transfer functions of the disturbance model and the real AVI's primary path

\section{Conclusions}

In this paper, we developed the process models of secondary path and primary path for an AVI system by employing the state space theory and NR-LMS algorithm. The validation was studied by comparing 
the FRFs from the identified model and the experiment data, and the models developed from identification experiment can match well with the real AVI system, which indicates the identify method employed in this paper is effective to build the dynamic MIMO model and it is accuracy enough for control application.

\section{Reference}

[1] Yun-Feng Wang, Wei Cheng, DESIGN AND ANALYSIS OF AN ACTIVE CONTROL PLATFORM FOR WORKING MACHINE ON SPACECRAFT, TEHNICKI VJESNIKTECHNICAL GAZETTE. 21, 4(2014), 815-824.

[2] Brij N Agrawal, Hong-Jen Chen, Algorithms for active vibration isolation on spacecraft using a Stewart platform, Smart Materials and Structures. 13 (2004) 873-880.

[3] L Vaillon, C Philippe, Passive and active micro-vibration control for very high pointing accuracy space system, Smart Materials and Structures. 8 (1999) 719-728.

[4] D. Kamesh, R. Pandiyan, Ashitava Ghosal, Modeling, design and analysis of low frequency platform for attenuating micro-vibration in spacecraft, Journal of Sound and Vibration. 329 (2010) 3431-3450.

[5] D. Kamesha, R. Pandiyan, Ashitava Ghosal, Passive vibration isolation of reaction wheel disturbances using a low frequency flexible space platform,Journal of Sound and Vibration. 331 (2012) 1310-1330.

[6] Wei-Yong Zhou, Guglielmo S. Aglietti, Zhe Zhang, Modelling and testing of a soft suspension design for a reaction/momentum wheel assembly. Journal of Sound and Vibration. 330 (2011) 4596-4610.

[7] Wei-Yong Zhou, Dong-Xu Li, Design and analysis of an intelligent vibration isolation platform for reaction/momentum wheel assemblies, Journal of Sound and Vibration. 331 (2012) 2984-3005.

[8] Gawronski W. K., Advanced structural dynamics and active control of structures[M]. Springer-Verlag Inc. , New York, 2004, pp. 219 - 226.

[9] Juang J. N. , Pappa R. S., An eigensystem realization algorithm(ERA) for model parameter identification and model reduction, Journal of Guidance , Control and Dynamics. 1985 , 8(5) 620 627.

[10] Cao Y , Chen X. B., State space system identification of 3-degreeof-freedom piezo-actuator-driven stages with unknown configuration, Actuators. 2 (2013) 1 - 18.

[11] De Callafon R. A. , Moaveni B., General realization algorithm for modal identification of linear dynamic systems, Journal of Engineering Mechanics. 134 (2008) 712 - 722.

[12] Wang Yunfeng, Cheng Wei, Chen Jiangpan, State Space Theory and NR-LMS Algorithm based Method for Structural Dynamics Parameter Identification, Journal of Beijing University of Aeronautics and Astronautics. 4 (2014) 517-522 (In Chinese).

[13] B. Bandyopadhyay, T. C. Manjunath, M. Umapathy, Modeling, Control and implementation of Smart Structures, Springer, 2007, 226-228.

[14] Wodek K , awronski. Advanced Structural Dynamics and Active Control of Structures, Springer-Verlag, Inc. , New York, 2004, pp.143-165. 